\title{
Assessing the extent of strategic manipulation: the average vote example
}

\author{
Régis Renault • Alain Trannoy
}

Received: 15 November 2010 / Accepted: 25 July 2011 / Published online: 21 September 2011 (C) The Author(s) 2011. This article is published with open access at SpringerLink.com

\begin{abstract}
The average voting procedure reflects the weighted average of expressed opinions in $[0,1]$. Participants typically behave strategically. We evaluate the discrepancy between the average taste and the average vote. If the population is sufficiently large, it is possible to construct approximations of both the average vote and the average taste which may be readily compared. We construct upper and lower bounds for the limit average vote that depend on the limit average taste. If the average taste is central enough, the range of possible values for the average voting outcome is narrower than the corresponding range for majority voting. For instance, if the average taste is at $1 / 2$, the limit equilibrium outcome is this value plus or minus roughly .2 , whereas the weighted median maybe anywhere in the $[0,1]$ interval.
\end{abstract}

Keywords Average voting $\cdot$ Nash equilibrium $\cdot$ Strategic bias $\cdot$ Weight

JEL Classification $\mathrm{D} 74 \cdot \mathrm{H} 41 \cdot \mathrm{I} 22$

We thank Hervé Moulin for a useful suggestion. We should also like to thank Francis Bloch, Alessandra Casella, Nicolas Gravel, Michel Le Breton and William Thomson and two referees for their comments on earlier versions of this paper. The usual caveat applies.

R. Renault

Université de Cergy-Pontoise, THEMA, 33 Bd du Port,

95011 Cergy-cedex, France

e-mail: regis.renault@u-cergy.fr

A. Trannoy $(\varangle)$

EHESS, GREQAM-IDEP, Vieille Charité, 2 rue de la Charité,

13002 Marseille, France

e-mail: alain.trannoy@univmed.fr 


\section{Introduction}

In response to the Gibbard-Satterthwaite theorem (Gibbard 1973; Satterthwaite 1975) much attention has been devoted to truthful implementation. Since his first published paper (Barbera 1977), Salvador Barbera has never stopped offering new deep insights of the requirement of strategy-proofness in a voting context (see his overview, Barbera 2006, 2011). Yet, strategy-proofness or less restrictive criteria, like Nash or Bayesian incentive compatibility, rule out some simple voting rules that are commonly used in practice as, for instance, plurality in political elections or Borda count in committee decisions. When dealing with such rules that allow for strategic manipulation, it is useful to assess the extent of the manipulation by performing a comparison of the outcome of strategic behavior with that of sincere voting. The latter is a natural benchmark since one might expect those who chose these rules at a constitutional stage to view them as appropriate modes of preference aggregation. Here we present a method for evaluating the extent of the distortion introduced by strategic manipulation, in other words the strategic bias, in the case of the average voting rule.

It is a very simple voting scheme that implements a weighted arithmetic mean of votes. Several countries have adopted procedures for allocating public funds, that may be described by a "forced to pay yet free to choose" mechanism. It turns out that the outcome of this mechanism can be regarded as a weighted average vote. In Spain, tax payers may earmark up to $0.5 \%$ of their income tax to the catholic church or to nongovernmental organisations and similar provisions can be found in Italy or Portugal. In Canadian provinces of Ontario and Saskatchewan, there are publicly financed separate school boards for Catholic schools along with the public school boards; households may choose which school system receives their property taxes. In France, high schools, colleges and universities are partly financed by a "training tax" that firms must pay, ${ }^{1}$ although they may decide on its allocation among different teaching institutions or training programs. Typically, firms and more particularly small ones choose to finance only one institution even if they can choose to subsidy as many institutions as they want. These tax mechanisms and more precisely the training tax ${ }^{2}$ are formally equivalent to weighted average voting rules. If there are only two possible uses of public funds, the vote of a tax payer is the fraction of her taxes that she chooses to allocate to one of them. Then the outcome of the vote (the proportion of public funds going to either use) is a weighted average of the votes, where the weight of each voter is her share in total tax contributions. Although the weights represent the individual shares in total wealth or in total tax contribution in all actual applications of the average vote that we are aware of, the interpretation of the weights may be broader. ${ }^{3}$ For instance,

\footnotetext{
1 Payrolls are taxed at a $0.5 \%$ rate, which yields a revenue of $€ 2$ billion in 2011 . In comparison, the revenues of the wealth tax are $€ 3$ billion. $22 \%$ of the budget of the second most well-known French business school, ESSEC, comes from this channel, whereas the proportion is still between 10 and $15 \%$ for other French business schools.

2 The vote itself is constrained to discrete values in some of the tax mechanisms to which we allude above. In this case, there is an important difference with the model presented in the paper where the choice interval varies in the interval $[0,1]$.

3 Average voting is here considered as a direct democracy mechanism. There are a number of papers in the political economy literature that describe the democratic political process as achieving a compromise
} 
if each voter represents a group (household, constituency, country ...), the weight may be the share of this group in the overall population.

Although there are numerous examples of its application, the average voting rule has only attracted limited attention. We know that sincere average vote yields an efficient outcome, if agent's preferences are Euclidean. In this case, the set of Pareto outcomes is identical to the set of weighted average votes. If there at least five agents, agents have Lipschitz utility functions and the voting space is multidimensional, the average voting rule is shown to be the unique anonymous and unanimous voting rule that satisfies a weakening of strategy-proofness in large voting problems (Ehlers et al. 2004). Bilodeau (1994) in his study of tax-earmarking institutions shows that leaving the spending decisions in the hands of tax payers yields a unique non-cooperative equilibrium in the core. Renault and Trannoy (2005a) exhibit circumstances where the average rule may be more suited to protect minorities than majority voting, taking into account the strategic behavior of voters. They also found that the equilibrium of the game in large population converges to the same fixed-point whatever the informational framework of the game, complete or incomplete (Renault and Trannoy 2005b). Marchese and Montefiori (2010) provide the first experiment of the manipulability of the average rule. They do not find a strong misrepresentation of preferences and the outcome of the game seems intermediate with respect to a sincere revelation of preferences and the outcome deriving from a strategic behavior.

The first objective of this paper is to check that the use of an average rule cannot be dismissed on the basis of a normative argument. To this end an axiomatic characterization of the average social choice rule is provided based on the idea that if the opinions of two individuals are moved in opposite directions with the same magnitude, the collective choice remains unchanged. Thus, contrary to the median, the mean allows for taking account of the magnitude in differences of opinions. However, even if one agrees that this is a desirable property there remains a problem with implementing such a rule. It is neither implementable in dominant strategy nor in a Nash equilibrium. The main part of the paper is devoted to characterizing the outcome of a voting procedure reflecting the average of expressed opinions when participants behave strategically, and to an evaluation of the magnitude of the strategic bias.

In the average voting game considered here, individuals choose an alternative in the $[0,1]$ interval and preferences are supposed to be single-peaked in order to allow an easy comparison of the outcome of the game with that of majority voting. In particular we compare the strategic bias in average voting with the discrepancy between the average and the median taste, which serves as a benchmark. Indeed, a distortion from the average taste that would exceed that obtained by using the strategy-proof majority rule would seem particularly unsatisfactory.

The game is studied in a complete information context. The agent's Nash equilibrium behavior is typically to vote either 0 or 1 , which is in tune with the empirical evidence for the training tax. The characterization of the equilibrium outcome is quite

Footnote 3 continued

modeled as a convex combination of the political platforms of the various parties (see Alesina and Rosenthal 1995, 1996). The weights depend on the distribution of votes among parties and there is therefore some formal equivalence with average voting, which is exploited in Gerber and Ortuno-Ortin (1998). 
clean and allows for an easy comparison with the outcome of a majority vote. It is less obvious how it relates to the average taste. However, if the population is sufficiently large, it is possible to construct approximations of both the average vote and the average taste that may be readily compared: the limit equilibrium allocation is characterized by a simple fixed point relation involving a function that, for all possible levels of the allocation $y$, indicates the expected relative weight of those who favor an outcome above $y$; the integral of this same function approximates the average taste for a large population.

These approximation results allow us to construct upper and lower bounds for the limit average vote that depend upon the limit average taste. Average voting prevents the outcome from being too extreme when the average opinion is central. It restricts the range of the social outcome more than the majority rule. For instance, if the average taste is at $1 / 2$, the limit equilibrium outcome is within plus or minus roughly 2 around this value, whereas the weighted median may lie anywhere in the $[0,1]$ interval. This result shows that in a very polarized society where a large number of voters have extreme opinions 0 or 1 , average voting is better suited than majority voting for achieving a compromise, i.e. an outcome different from the most extreme opinions. ${ }^{4}$ Moreover, the strategic bias is at most .21, again reached for an average taste of one half. When the average taste is closer to one of the boundaries of the choice space, namely the average taste is smaller than $1 / 4$ or larger than $3 / 4$, the strategic bias may be more important than the gap between the average and the weighted median taste. Indeed, the strategic power is much stronger for those who favor an outcome in the center of the choice space; in this case they are all located on one side of the average taste.

Section 2 presents an axiomatization of the weighted average voting rule in order to shed some light on its normative properties. We gather what is known about the average voting game in the following section. A limit approximation of the average taste is provided in Sect. 4, as well as the main results regarding the approximation of the Nash outcome in a complete information setting for large populations, the strategic bias and the comparison with majority voting. Section 5 concludes. Proofs of results are gathered in the appendix.

\section{Normative foundations}

The social choice problem under consideration is as follows. The social state $y$ belongs to the interval $[0,1]$. There are $n$ individuals indexed by $i$. Each voter's preferences, $R_{i}$, are single-peaked with $b\left(R_{i}\right)$ denoting the bliss point and are represented by a continuous utility function, $u_{i}$. The set of continuous single-peaked preferences on $[0,1]$ is denoted $S P$. Each individual, $i$ is endowed with a relative weight, $w$, where $w \equiv\left(w_{1}, \ldots, w_{n}\right)$ is an element of the $(n-1)$-dimensional simplex $S^{n-1}$. The social decision depends upon individual weights as well as preferences and is defined for

\footnotetext{
${ }^{4}$ See Border and Jordan (1983), for a related formulation of the uncompromising nature of the median rule. The majority rule does not exploit the continuity of the choice space which would allow for such a compromise.
} 
any population size. A social choice rule is a mapping $f: \cup_{n=1}^{\infty} S P^{n} \times S^{n-1} \rightarrow[0,1]$. An example of such a rule is the weighted average social choice rule which is defined as follows.

Definition 2.1 The weighted average social choice rule $f_{w a}$ is defined by:

$$
\forall n, \forall(R, w) \in S P^{n} \times S^{n-1}, f_{w a}(R, w)=\sum_{i=1}^{n} w_{i} b\left(R_{i}\right)
$$

This section aims at providing an axiomatic characterization of this rule. For further reference, we define the anonymous average social choice rule.

Definition 2.2 The average social choice rule with equal weights $f_{a}$ is defined by:

$$
\forall R \in S P^{n} f_{a}(R)=\frac{1}{n} \sum_{i=1}^{n} b\left(R_{i}\right)
$$

The first axiom is standard.

Definition 2.3 The social choice rule $f: \cup_{n=1}^{\infty} S P^{n} \times S^{n-1} \rightarrow[0,1]$ is unanimous if

$$
\begin{array}{r}
\forall(R, w) \in \cup_{n=1}^{\infty} S P^{n} \times S^{n-1} \text { with } w_{i}=\frac{1}{n} \forall i, f(R, w)=b\left(R_{1}\right) \\
\text { whenever } R_{i}=R_{1} \forall i=1, \ldots, n .
\end{array}
$$

Unanimity is a weakening of Pareto efficiency. In order to single out the average rule among unanimous rules, we introduce an axiom which has a natural interpretation in the context of voting procedures.

Definition 2.4 The social choice rule $f: \cup_{n=1}^{\infty} S P^{n} \times S^{n-1} \rightarrow[0,1]$ satisfies cancellation of opposite changes in preferences if

$$
\begin{aligned}
& \forall(R, w),\left(R^{\prime}, w\right) \in \cup_{n=1}^{\infty} S P^{n} \times S^{n-1} \text { with } w_{i}=\frac{1}{n} \forall i, f(R, w)=f\left(R^{\prime}, w\right) \\
& \text { whenever } b\left(R_{j}^{\prime}\right)-b\left(R_{j}\right)=b\left(R_{k}\right)-b\left(R_{k}^{\prime}\right) \text { for some } j \text { and } k \text {, and } \\
& b\left(R_{i}\right)=b\left(R_{i}^{\prime}\right) \forall i \neq j, k .
\end{aligned}
$$

In words, this axiom requires that the social choice is unaffected if two individuals change opinions so that the moves are in opposite directions and have the same magnitude. It turns out that this independence leads to the additivity property of the social choice function. We now discuss its interpretation in relation with the Cancellation axiom introduced by Ching. ${ }^{5}$ Ching's condition may be stated as follows.

\footnotetext{
5 Ching (1995) has introduced this axiom in a characterization of the median social choice rule.
} 
Definition 2.5 A social choice rule $f: \cup_{n=1}^{\infty} S P^{n} \times S^{n-1} \rightarrow[0,1]$ satisfies cancellation of extreme preferences if, for any set of $n$ voters, for any profile of preferences, $R \in S P^{n}$, for any $R_{n+1}$ and $R_{n+2}$ in $S P$, if $b\left(R_{n+1}\right)=0$ and $b\left(R_{n+2}\right)=1$, then $f\left(R, R_{n+1}, R_{n+2} ; \frac{1}{n+2}, \ldots, \frac{1}{n+2}\right)=f\left(R ; \frac{1}{n}, \ldots, \frac{1}{n}\right)$.

This condition says that two additional voters should offset each other if they hold opposite extreme opinions.

Typically a non dictatorial social choice rule strikes a balance between divergent opinions. The two cancellation axioms provide two different approaches to how this balancing act should be performed. Both deal with a symmetric change in the tastes of the population. In the Ching axiom, the symmetry relates to the position of the new members relative to the social choice. A bliss point at 0 is always below the social choice while a bliss point at 1 is always above. All that is required for making such statements is that the set of alternatives is ordered. In the axiom of cancellation of opposite changes in preferences the symmetry relates to the moves in bliss points. They must be in opposite directions and have the same magnitude. This condition exploits the normed linear space structure of the set of social alternatives.

It can be shown that the first cancellation axiom along with unanimity are sufficient to characterize the average rule with equal weights. ${ }^{6}$ One interesting consequence of this result is that for unanimous social choice rules and single-peaked preferences, the two cancellation axioms are incompatible with each other since the unweighted average voting rule does not satisfy cancellation of extreme preferences. Thus the choice between the two principles cannot be avoided.

In order to tackle the characterization of the non anonymous average rule it is necessary to introduce additional axioms. In particular, we need to be more specific about the role of individual weights in the collective decision. To do this we introduce a principle according to which the social choice should only depend on the weighted distribution of bliss points. In particular, for two populations with different sizes, if this distribution is identical, then the social choice should be identical. Formally, we define the weighted cumulative distribution, $L$, associated to a preference and weight profile $(R, w) \in \cup_{n=1}^{\infty} S P^{n} \times S^{n-1}$ by

$$
L(b: R, w)=\sum_{i \mid b_{i} \leq b} w_{i}, \quad \forall b \in[0,1] .
$$

We now introduce the distribution invariance axiom.

Definition 2.6 A social choice rule $f$ satisfies the distribution invariance axiom if for any $(R, w),\left(R^{\prime}, w^{\prime}\right) \in \cup_{n=1}^{\infty} S P^{n} \times S^{n-1}$, if $L(.: R, w)=L\left(.: R^{\prime}, w^{\prime}\right)$, then $f(R, w)=f\left(R^{\prime}, w^{\prime}\right)$.

\footnotetext{
6 In the location literature, Holzman (1990) proposes an axiom that singles out the equal weight average rule among all unanimous rule. To quote the author, "It is a Lipschitz condition which can be understood as a strong type of continuity requirement: the solution should not be too sensitive to small changes in the data (perhaps due to errors of measurement)".
} 
To illustrate the relevance of this axiom, ${ }^{7}$ let us consider the example of the US senate where each senator represents half a state. Suppose that the weight of each senator is given by the relative share of his constituency in the US population. Now suppose that we switch to direct democracy in which each American has the same weight and that in each constituency, the whole population expresses the same opinion as that of its senator. Then the axiom requires that the social choice should be unchanged.

The statement of our first result requires a technical continuity axiom which is the following.

Definition 2.7 A social choice rule $f$ is weight-continuous if, for any $n$, for any $R \in S P^{n}, f(R,$.$) is continuous in w$ on $S^{n-1}$.

It is easily checked that the four axioms, unanimity, cancellation of opposite changes in preferences, distribution-invariance and weight-continuity are mutually independent. $^{8}$

The following proposition provides an axiomatic characterization of the weighted average social choice rule. ${ }^{9}$

Proposition 2.1 A social rule $f: \cup_{n=1}^{\infty} S P^{n} \times S^{n-1} \rightarrow[0,1]$ is weight continuous, unanimous, distribution-invariant and satisfies cancellation of opposite changes of preferences if and only if it is the weighted average rule $f_{w a}$.

If the average rule is selected we are left with the problem of implementation since it is clearly not strategy-proof. Furthermore it is not Nash implementable since it does not satisfy the monotonicity criterion of Maskin (1985). This raises the question of what would be the social decision if, in spite of these implementation difficulties, the average rule was applied to expressed opinions rather than to actual ones. This issue is addressed in subsequent section, taking account of the potential strategic behavior of voters.

\section{The equilibrium outcome}

We start with a brief description of the average voting game along with an overview of existing results. Henceforth notation is simplified so that $b_{i}$ denotes voter $i$ 's bliss point. There are $n$ voters with singlepeaked preferences over the choice space which is the unit interval. Each voter $i$ chooses a vote denoted $s_{i}$ in $[0,1]$ and voting involves no costs. Agents cast their votes simultaneously. The allocation is then defined by

$$
y=\sum_{i=1}^{n} w_{i} s_{i},
$$

\footnotetext{
${ }^{7}$ Clearly, the axiom is only meaningful if the weights emerge from a normative analysis.

${ }^{8} f_{1}()=.\left[\sum_{i} w_{i} b\left(R_{i}\right)\right]^{2}$ satisfies all axioms but unanimity. $f_{2}()=.\sqrt{\sum_{i} w_{i} b\left(R_{i}\right)^{2}}$ satisfies all axioms but cancellation. $f_{3}()=.f_{a}$ satisfies all axioms but invariance. $f_{4}()=.f_{w a}$ for $w_{i}$ rational for all $i, f_{4}()=.f_{2}$ for $w_{i}$ irrational for some $i$ satisfies all axioms but weight-continuity.

9 Aczel and Wagner (1981) offer an axiomatic characterization of the set of all possible weighted average rules where there is no a-priori specification of the weights. Their result however does not apply to a one dimensional problem.
} 
where $w_{i} \geq 0$ is the relative weight of voter $i$, for any $i$, and $\sum_{i=1}^{n} w_{i}=1$.

To understand how agents behave in a Nash equilibrium, it is useful to describe best responses. Other player's choices are only relevant to player $i$ through an aggregate vote. Let $S_{-i}$ be the weighted sum of votes by voters other than $i$, that is, $S_{-i}=$ $\sum_{j \neq i} w_{j} s_{j}$. Then agent $i$ 's best response is defined by

$$
s_{i}\left(b_{i}, S_{-i}\right)= \begin{cases}1 & \text { if } b_{i}-S_{-i}>w_{i} \\ \frac{b_{i}-S_{-i}}{w_{i}} & \text { if } 0 \leq b_{i}-S_{-i} \leq w_{i} \\ 0 & \text { if } b_{i}-S_{-i}<0\end{cases}
$$

If the aggregate vote by others is below the bliss point $b_{i}$ two situations are possible, depending on the size of the discrepancy. If it is larger than agent $i$ ' $\mathrm{s}$ weight, it is optimal to pick the largest possible vote which is 1 . If the difference is smaller, agent $i$ 's weight in the average vote, $w_{i}$, enables him to make up for the discrepancy, in which case he obtains his exact bliss point as the final outcome. If the aggregate vote by others yields a value that is above the bliss point, it is optimal to vote 0 since any non zero vote would make the situation worse.

The characterization of the equilibrium outcome requires that individuals be ranked according to decreasing values of $b_{i}$. Let us define:

$$
W_{i}=\sum_{j=1}^{i} w_{j}
$$

Now let

$$
i^{*}=\min \left\{i \in\{1, \ldots, n\} ; W_{i} \geq b_{i+1}\right\} \quad \text { with } b_{n+1}=0 .
$$

In order to state existing results regarding the equilibrium of the average voting game, we first give two definitions. We define the median of a finite set of real numbers $A$ with $N$ elements as the smallest number $\operatorname{med}(A) \in A$ that satisfies

$$
\frac{1}{N} \#\{a \in A: a \leq \operatorname{med}(A)\} \geq \frac{1}{2} \text { and } \frac{1}{N} \#\{a \in A: a \geq \operatorname{med}(A)\} \geq \frac{1}{2}
$$

If $N$ is odd, condition (3) defines a unique number while if it is even, there are 2 such numbers. We adopt the convention that the median is the smallest.

We also define a strong Nash equilibrium. Let $C$ be a coalition i.e. a subset of the set of voters, and let $\bar{C}$ the complement of $C$ in the set of voters. The $n$-tuple $s^{*}=\left(s_{1}^{*}, \ldots, s_{n}^{*}\right)$ is a pure-strategy strong Nash equilibrium if for all coalitions $C$ and for all $s \in[0,1]^{n}$,

$$
u_{i}\left(\sum_{j} w_{j} s_{j}^{*}\right) \geq u_{i}\left(\sum_{j \in C} w_{j} s_{j}+\sum_{j \in \bar{C}} w_{j} s_{j}^{*}\right) \quad \text { for all } \mathrm{i} \in C
$$


where $u_{i}$ denotes voter $i$ 's utility. The above expression means that the $n$-tuple $s^{*}$ is a best response for any coalition of players.

Proposition 3.1 The average voting game has a Nash equilibrium. The Nash equilibrium allocation is unique and may be described by the following two equivalent formulas:

$$
\begin{gathered}
y^{*}=\min \left\{b_{i^{*}}, W_{i^{*}}\right\} . \\
y^{*}=\operatorname{med}\left(b_{1}, \ldots, b_{n}, W_{1}, \ldots, W_{n-1}\right)
\end{gathered}
$$

\section{Furthermore any Nash equilibrium is strong Nash.}

The two characterizations are established in Renault and Trannoy (2005a) and the argument showing that any Nash equilibrium of this game is strong Nash can be found in Bilodeau (1994). ${ }^{10}$ Since, as Bernheim et al. (1987) point out, any Strong Nash equilibrium is coalition-proof, any Nash equilibrium of this game is also coalitionproof. The first characterization sheds light on the cut-off point represented by the bliss point of individual $i^{*}$. She is able to obtain her preferred option if it is smaller than the cumulative wealth of individuals whose preferred option is on her left on the unit interval.

It is quite uncommon that a strategic reasoning leads to a formula that can be obtained through a normative analysis. Indeed, characterization (ii) bears a striking resemblance with the extended median of Moulin (1980) ${ }^{11}$ who shows that a social choice rule, $f$, is peak-only (i.e. the sole relevant information about preferences for the social choice rule is the list of bliss points), strategy-proof, anonymous and efficient if and only if there exist $n-1$ parameters in $[0,1], a_{1}, \ldots, a_{n-1}$ such that for all profiles of single peaked preferences, $R$,

$$
f(R)=\operatorname{med}\left\{b\left(R_{1}\right), \ldots, b\left(R_{n}\right), a_{1}, \ldots, a_{n-1}\right\} .
$$

In this definition, the parameters $a_{1}, \ldots, a_{n-1}$ are independent of the preference profile under consideration while the parameters $W_{1}, \ldots, W_{n-1}$ typically depend on how the bliss points are ranked. Note that, however, if weights are identical, characterization (ii) is an extended median with parameters $\left\{\frac{1}{n}, \frac{2}{n}, \ldots, \frac{n-1}{n}\right\}$ no matter what the ordering of bliss points turns out to be. To our knowledge this voting procedure provides the first illustration of how the parameters of an extended median may have an economic interpretation, namely as population shares. Here the uniform distribution corresponds to an equal treatment of individuals. The careful reader will notice

\footnotetext{
10 The uniqueness result is reminiscent of Gerber and Ortuno-Ortin (1998) who find, in a similar game with a continuum of agents that there exists a unique strong Nash equilibrium in which voters use cut points strategies. The unique strong Nash allocation is also a core allocation, when the core definition keeps in line with the literature on voting games with the majority rule (see for instance Ordeshook 1986).

11 See also Sprumont (1995) for a detailed survey of this material.
} 
that the parameters $\left\{\frac{1}{n}, \frac{2}{n}, \ldots \frac{n-1}{n}\right\}$ are related to the outcome of the social choice for some specific profiles of preferences. More precisely it is easily checked that $\frac{n-i}{n}$ is the outcome of the extended median rule when $n-i$ (respectively $i$ ) individuals have 1 (resp. 0) as bliss points. It is also the average taste for this profile. Since, in this case, no individual wants to manipulate his vote when faced with the average voting procedure, the outcome of this procedure will coincide with the true mean of bliss points. The same kind of remarks applies for the non-anonymous case at the price of some additional complexity.

We now turn to our main focus, which is an evaluation of how much strategic behavior distorts the outcome of the vote from the average taste. To this end, the remainder of the paper considers the average voting game with a large population of voters. For the following, the reader needs to remember that in equilibrium, all those with bliss points strictly above the equilibrium allocation vote 1 while all those with bliss points strictly below the equilibrium allocation vote 0 .

\section{Assessing the strategic bias with a large population of voters}

\subsection{Inferring the average taste from aggregate data}

Although we assume in this section that weights and bliss points are common knowledge for the voters, this common knowledge may not be shared by an outside observer. He may only have some aggregate knowledge, namely, he does not know any more than the joint probability distribution of bliss points and weights. From this point of view, it is relevant to provide an approximation of the equilibrium outcome which requires only the knowledge of this probability distribution when the population is large enough. Correlation between bliss points and weights is allowed since it may be present in all practical applications.

To proceed with the limit argument, we need to derive a simple expression for the limit of the weighted average taste. Bold characters denote random variables. A vote with $n$ participants is given by $n$ independent draws from a probability distribution $\mathcal{P}$ defined on $[0,1] \times \mathcal{R}_{++}$admitting a continuous density. For each player $i$, the first component is his bliss point $\mathbf{b}_{i}$ and the second component is his absolute weight $\omega_{i}$, which contrary to relative weight $w_{i}=\omega_{i} / \sum_{i=1}^{n} \omega_{i}$ is not restricted to be in $[0,1] .{ }^{12}$ Let $F$ denote the marginal c.d.f. of $\mathbf{b}_{i}$ and let $J\left(. \mid b_{i}\right)$ be the conditional c.d.f. of $\omega_{i}$. We further assume that the conditional distributions have finite mean for all values of conditioning variables. Let $\mu(b)$ denote the conditional mean and $\bar{\mu}$ denote the unconditional mean of $\omega_{i}$. We now define the decreasing function $H$ on $[0,1]$ as follows

$$
H(y)=\bar{\mu}^{-1} \int_{y}^{1} \int_{0}^{+\infty} \omega d J\left(\omega \mid b_{i}\right) d F\left(b_{i}\right)=\bar{\mu}^{-1} \int_{y}^{1} \mu\left(b_{i}\right) d F\left(b_{i}\right) .
$$

\footnotetext{
12 Specifying absolute weights is convenient because, contrary to relative weights, they may be drawn independently, and we may therefore resort to the law of large numbers in the proofs.
} 
This function is decreasing from $H(0)=1$ to $H(1)=0$. It measures the expected relative cumulative weight of individuals with bliss points in excess of $y$. In the special case where weights are independent from bliss points, we have $H(y)=1-F(y)$.

In the general case, the function $H$ may be also related to $F$ thanks to a concentration curve. Whenever we plot shares of a variable $X$ against quantiles in the distribution of a variable $Y$, the result is called a concentration curve for $X$ with respect to $Y$. Now define $G$ as the function that, for all $y \in[0,1]$, maps $1-F(y)$ into $H(y)$ so that

$$
H(y)=G(1-F(y)) .
$$

Note that $1-F(y)$ is the expected cumulative proportion of the population with bliss points above $y$, while $H(y)$ is the expected cumulative relative weight of this subpopulation. The function $G$ may therefore be viewed as the concentration curve for weights with respect to bliss points.

The function $H$, which plays a critical role in the paper, may first be used to derive a simple characterization of the limit of the average taste as the population becomes large.

Claim 4.1 The weighted average taste for $n$ voters $v_{n}=\sum_{i=1}^{n} w_{i} b_{i}$ converges to $v=\int_{0}^{1} H(b) d b$ with probability 1 when $n$ goes to infinity.

This result generalizes the well known formula for the expected value of a random variable with c.d.f. $F$ as $\int_{0}^{1}(1-F(t)) d t$. The generalization we consider here allows for different realizations of a random variable to be weighted differently.

As we now show, the function $H$ may also be used to construct an approximation for the Nash outcome of average voting.

\subsection{Inferring the Nash outcome from aggregate data}

Let $F_{n}, J_{n}\left(. \mid b_{i}\right), \mu_{n}\left(b_{i}\right), \overline{\mu_{n}}$ and $H_{n}(y)$ be the empirical counterparts of $F, J\left(. \mid b_{i}\right)$, $\mu\left(b_{i}\right), \bar{\mu}$ and $H(y)$ for the $n$-players game. It is readily shown that

$$
H_{n}(y)=\bar{\mu}_{n}^{-1} \int_{y}^{1} \mu_{n}\left(b_{i}\right) d F_{n}\left(b_{i}\right)=\frac{\sum_{i=1}^{n} \omega_{i} I\left(b_{i} \geq y\right)}{\sum_{i=1}^{n} \omega_{i}},
$$

so that it measures the relative cumulative weight of agents with bliss points of at least $y$ (where $I$ denotes an indicator function). This empirical cumulative weights function $H_{n}$ is a decreasing step function which is left-continuous. Points of discontinuity correspond to realized bliss points $\left\{b_{i}\right\}_{i=1}^{n}$ and the jump at $b_{i}$ measures the relative weight of individuals with bliss point $b_{i}$. From now on, $\mathbf{y}_{n}^{*}$ denotes the equilibrium allocation when the population size is $n$ and $y^{*}$ denotes the unique solution to

$$
y^{*}=H\left(y^{*}\right)=G\left[1-F\left(y^{*}\right)\right] .
$$

Proposition 4.1 The sequence $\left\{\mathbf{y}_{n}^{*}\right\}$ converges to $y^{*}$ with probability 1 . 
In the limit, votes are concentrated at the extremes, 0 or 1 , so that the outcome is given by the cumulative weight of those voting $1, H\left(y^{*}\right)$, and it is also equal to the bliss point of the pivotal individual, $y^{*}$.

An interesting special case of the above result is when weights $\omega_{i}$ are independent of bliss points $b_{i}$. Then, $\mu\left(b_{i}\right)=\bar{\mu}$ for all $b_{i}$. Here the game is anonymous in expectation in the sense that the expected weight of individuals is the same no matter what their tastes might be. Therefore, from (4), $H(y)=1-F(y)$. Thus $y^{*}$ is defined by the simple fixed point relation

$$
y^{*}=1-F\left(y^{*}\right) .
$$

The approximations that we have derived for the average taste and the Nash outcome are quite simple and may be used to evaluate the extent of the strategic bias.

\subsection{Strategic bias}

We now investigate what are the boundaries on the deviation from the average bliss point caused by strategic voting. To this end we establish the following proposition pertaining to the sign and magnitude of the strategic bias, i.e. the gap between the average voting outcome and the average taste $v=\int_{0}^{1} H(b) d b$.

Proposition 4.2 (i) For any $H, 1-\sqrt{1-v} \leq y^{*} \leq \sqrt{v}$.

(ii) Furthermore, if $H$ is strictly convex, then $y^{*} \geq v$, while if $H$ is strictly concave the inequality is reversed.

The first result provides bounds on the value of the Nash outcome as a function of the average taste. As shown by the proof of the above proposition, the obtained bounds are tight in the sense that it is always possible to specify a continuous function $H$ such that the average voting outcome is arbitrarily close to one of these bounds. The largest possible interval is obtained for a mean of $1 / 2$, in which case the lowest possible Nash outcome is $1-\sqrt{1 / 2}$ and the largest is $\sqrt{1 / 2}$. Thus, average voting guarantees that if the average taste is moderate, the collective choice cannot be too extreme. In this particular configuration, the allowed interval for the average vote outcome is symmetric with respect to the average taste, a property that is lost when the average taste is closer to 0 or 1 . Indeed, players who have an opinion farthest from the boundaries of the choice space can pull the outcome towards their preferred opinion very effectively, since they may hugely distort their expressed opinion from the true one by casting an extreme vote. Thus, for instance, if the average taste is below $1 / 2$, the largest potential upward bias is greater than the largest potential downward bias.

The second result provides some hint as to how the sign of the strategic bias is related to the skewness of the weighted distribution of bliss points: concavity or convexity of $H$ corresponding to the extreme cases of a monotonically increasing or decreasing density. If the distribution is skewed to the right so that the top part of $H$ tends to be convex, the bias should be expected to be upwards. This is because the mean is close to zero so that those who favor an outcome below the mean have only a limited ability to distort their vote; the most they can do is to vote zero. By contrast, those who favor 


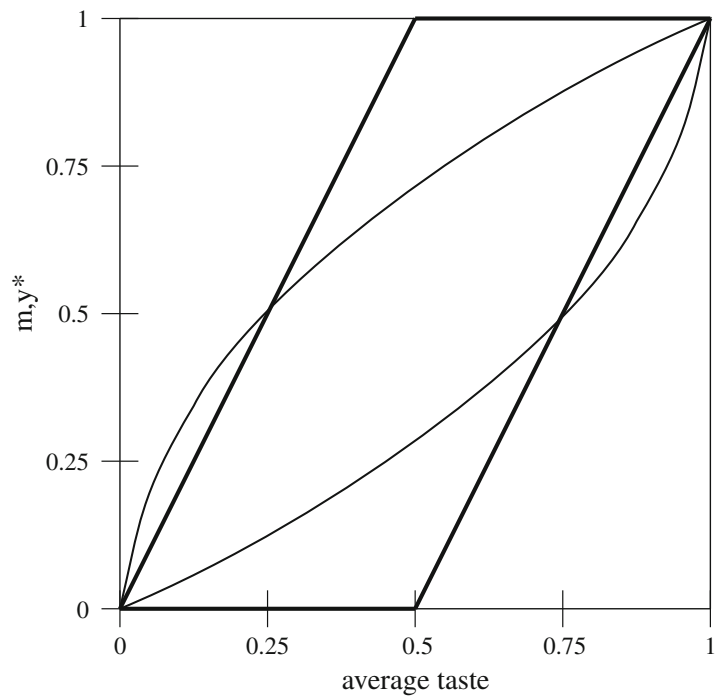

Fig. 1 The thick lines (resp. thin lines) represents the allowed interval for the majority (resp. average) outcome in function the average taste

an outcome above the mean have bliss points remote from 1 so that they may hugely exaggerate their taste.

Since the set of all allowed values for the average voting outcome is fairly large, one may wonder whether it is narrower than the set of allowed values for the outcome of the majority vote. Here, the relevant majority vote is one where each voter is weighted as in the weighted average taste, so that the outcome is a weighted median $m_{n}$ defined as follows

$$
\sum_{i / b_{i} \leq m_{n}} w_{i} \geq \frac{1}{2} \quad \text { and } \sum_{i / b_{i} \geq m_{n}} w_{i} \geq \frac{1}{2}
$$

with the convention that if there are two such numbers the smallest will be selected.

It is straightforward to establish that when $n$ converges to infinity, $m_{n}$ converges with probability 1 to $m$ defined by

$$
H(m)=1 / 2 .
$$

The proof of the following proposition is similar to the proof of (i) in the previous proposition.

Proposition 4.3 $\operatorname{Max}(0,2 v-1) \leq m \leq \operatorname{Min}(1,2 v)$.

The comparison of the intervals in Propositions 4.2(i) and 4.3 leads to mixed conclusions about the merits of average voting relatively to the weighted majority vote in reflecting the average taste. It is readily verified (see Fig. 1 for an illustration) that the allowed interval for the average outcome is strictly included in that for the weighted median as long as the average taste is strictly between $1 / 4$ and 3/4. The advantage of 
the average voting rule over majority voting is especially telling in this case, since the magnitude of the interval and therefore of the potential discrepancy between the average taste and the outcome of the vote is maximal. For a smaller average taste, both the lower and upper bound on $y^{*}$ are strictly above the bounds on the median. The opposite configuration prevails for an average taste above 3/4. In these two cases, neither interval is a subset of the other. ${ }^{13}$ Then, as the average taste approaches either end point, no clear-cut conclusion about the relative merit of the average vote may be drawn. Nevertheless, the potential distortion from the average taste is less of an issue as the average gets closer to one end, since the allowed intervals for both the average vote and the majority vote outcomes become smaller. ${ }^{14}$

We conclude that if the average taste is located close to 0 or 1 , the average voting rule is hampered by the excessive strategic power of voters whose bliss points are in the center. One way to remedy this is to limit the set of possible votes as is done in actual applications of the average voting rule in Italy, Portugal and Spain.

Finally it should be noted that a comparison of the allowed interval for the two possible outcomes is relevant only if the policy-maker has a poor information about the underlying distribution of bliss points and weights that generates the function $H$. It may well be that, for a specific distribution, the outcome of the average vote does worse than the outcome of majority voting in approximating the average taste even though the latter falls in [1/4, 3/4]. Consider for instance the case of a population comprised of two subgroups: $60 \%$ are drawn according to a continuous uniform probability distribution on the support $[0, .4]$ and there is a mass point of $40 \%$ at .4 . The outcome with the average vote is .4 , whereas the weighted median is about .33 which is closer to the mean 28 .

\section{Conclusion}

The average voting rule is an example of a voting procedure that is used in various contexts even though it is not immune to strategic manipulation. Typically, voters choose to cast extreme votes. Our focus here is on the extent of this strategic manipulation as it is reflected in the discrepancy between the outcome of the vote and the average taste. The outcome of the average voting game may easily be compared with the mean of the populations' true opinions when the population is large enough. It is possible to establish that the outcome of average voting lies in some interval containing the average taste. The strategic bias may then be evaluated by comparing this interval with the range of potential outcomes for majority voting. If the average taste is not too extreme, then the range of potential outcomes for average voting is included in the corresponding range for majority voting. For more extreme average tastes, neither voting procedure dominates the other but they both yield outcomes that may not be too remote from the average taste.

\footnotetext{
13 It can be mentioned that the length of the interval for the average vote is smaller than that of the weighted majority vote for $v \in[0.157,0.843]$.

14 This, however, is not true in relative terms since the ratio of the largest possible average vote outcome to the mean tends to infinity as the mean tends to zero.
} 
In all the analysis, whatever it is normative or strategic, weights are given. It is clear that more work is needed to make weights part of the design of the mechanism. It is a possible avenue for further research.

The more general message that we wish to convey is that, although truthful implementation is a desirable property for a social choice rule, imposing such a requirement may lead to an excessive impoverishment of social choice theory. A different approach exemplified by this paper would be to compare the relative merits of the outcomes of different voting procedures whatever their strategic properties may be.

Open Access This article is distributed under the terms of the Creative Commons Attribution License which permits any use, distribution and reproduction in any medium, provided the original author(s) and source are credited.

\section{A Appendix}

\section{A.1 Proof of Proposition 2.1}

It is straightforward to check that the weighted average rule satisfies all axioms. It is now shown that is the only one.

First note that cancellation of opposite changes in preferences implies that the social choice rule is peak-only, i.e. $\forall R, R^{\prime} \in S P^{n}$ with $w=w^{\prime}, f(R)=f\left(R^{\prime}\right)$ whenever $b\left(R_{i}\right)=b\left(R_{i}^{\prime}\right) \forall i=1, \ldots, n$.

It is first shown that for rational weights, the first three axioms uniquely define $f_{w a}$. Consider a population of size $n$ for which preferences and weights are given by $(R, w)$ where $w$ is comprised of rational numbers. It is straightforward to show that there exists a population with size $n^{\prime}$ with preferences and weights given by $\left(R^{\prime}, \frac{1}{n^{\prime}}, \ldots, \frac{1}{n^{\prime}}\right)$ such that $L(.: R, w)=L\left(.: R^{\prime}, \frac{1}{n^{\prime}}, \ldots, \frac{1}{n^{\prime}}\right)$.

Let $R^{a} \in S P^{n^{\prime}}$ be a profile such that $b\left(R_{i}^{a}\right)=f_{a}\left(R^{\prime}\right), \forall i=1, \ldots, n^{\prime}$. By the theorem of Hardy, Littlewood and Polya theorem (1952) used in inequality measurement, there exists a finite sequence of preference profiles $\left(R^{1}, \ldots, R^{\ell}, \ldots, R^{m}\right)$ with $R^{1}=R^{\prime}$ and $R^{m}=R^{a}$ with the following property: for all $\ell<m$, there exist $j$ and $k$ such that $b\left(R_{i}^{\ell}\right)=b\left(R_{i}^{\ell+1}\right) \forall i \neq j, k$ and $b\left(R_{j}^{\ell+1}\right)-b\left(R_{j}^{\ell}\right)=b\left(R_{k}^{\ell}\right)-b\left(R_{k}^{\ell+1}\right)$. By cancellation of opposite changes, we must have $f\left(R^{\prime}\right)=f\left(R^{a}\right)$. Peak-only and unanimity imply that $f\left(R^{a}\right)=f_{a}\left(R^{\prime}\right)$. Thus $f\left(R^{\prime}\right)=f_{a}\left(R^{\prime}\right)$. Now, from distribution-invariance, $f\left(R^{\prime}, \frac{1}{n^{\prime}}, \ldots, \frac{1}{n^{\prime}}\right)=f(R, w)$ Since $f_{a}\left(R^{\prime}\right)=f_{w a}(R, w)$, we have $f(R, w)=f_{w a}(R, w)$.

Since rationals are dense in the real line, weight-continuity allows to show that the result holds for any vector of real weights.

\section{A.2 Proof of Claim 4.1}

We have $\bar{b}_{n}=\frac{\sum_{i=1}^{n} \omega_{i} b_{i}}{\sum_{i=1}^{n} \omega_{i}}$. The top expression has expectation $n \int_{0}^{1} b \mu(b) d F(b)$ which, integrating by parts and using (4) in the text is equal to $n \mu \int_{0}^{1} H(b) d b$. Since $\sum_{i=a}^{n} \omega_{i}$ has expectation $n \mu$, the result follows from the strong law of large numbers. 


\section{A.3 Proof of Proposition 4.1}

For any $y \in[0,1]$, let $H_{n}(y+)$ denote the limit on the right of $H_{n}$ at $y$ which corresponds to the cumulative weight of individuals with bliss points strictly exceeding $y$. Since in equilibrium, those with bliss points strictly above $y_{n}^{*}$ vote 1 and only those with bliss points of at least $y_{n}^{*}$ may have a strictly positive vote,

$$
H_{n}\left(y_{n}^{*}+\right) \leq y_{n}^{*} \leq H_{n}\left(y_{n}^{*}\right) .
$$

Thus if $\left\{\mathbf{H}_{n}\right\}$ converges uniformly to $H$ with probability 1 , since $H$ is continuous, $\left\{\mathbf{y}_{n}\right\}$ must converge to $y^{*}$ with probability 1 . In the remainder of the proof, we establish the uniform convergence of $\left\{\mathbf{H}_{n}\right\}$ to $H$ with probability $1 .{ }^{15}$

Let us first rewrite $\mathbf{H}_{n}(y)$ as

$$
\mathbf{H}_{n}(y)=\frac{\frac{\sum_{i=1}^{n} \omega_{i} I\left(b_{i} \geq y\right)}{n}}{\frac{\sum_{i=1}^{n} \omega_{i}}{n}}
$$

where $I$ is an indicator function. Random variables $\omega_{i} I\left(b_{i} \geq y\right)$ are drawn independently from an identical distribution with mean $\int_{y}^{1} \mu(b) d F(b)$. Applying the strong law of large numbers to $\left\{\omega_{i} I\left(b_{i} \geq y\right)\right\}$ and $\left\{\omega_{i}\right\}$ yields that $\left\{\mathbf{H}_{n}(y)\right\}$ converges to $H(y)$ with probability 1 for all $y$ rational. Thus these countably many events are true with probability 1 .

Finally, standard arguments may be used to establish that since $H_{n}$ is monotonically decreasing on $[0,1]$ for all $n$ and $H$ is continuous on $[0,1]$, pointwise convergence of $\left\{H_{n}\right\}$ to $H$ for a dense subset of $[0,1]$ containing 0 and 1 , implies uniform convergence on $[0,1]$. $^{16}$

\section{A.4 Proof of Proposition 4.2}

To prove (i), let us show that

$$
y^{* 2} \leq v \leq 2 y^{*}-y^{* 2}
$$

which clearly implies (i).

We have

$$
v=\int_{0}^{1} H(x) d x=\int_{0}^{y^{*}} H(x) d x+\int_{y^{*}}^{1} H(x) d x .
$$

\footnotetext{
15 The following proof is adapted from that of Lemma 2 in Goldie (1977).

16 See Lemma 1 in Goldie (1977) for an analogous result.
} 
Using the definition of $y^{*}$, since $H$ is decreasing, $H$ is bounded below by the step function taking a value of $y^{*}$ on $\left[0, y^{*}\right]$ and 0 on $\left(y^{*}, 1\right]$. Similarly, it is bounded above by the step function taking a value of 1 on $\left[0, y^{*}\right]$ and $y^{*}$ on $\left(y^{*}, 1\right]$. The result follows.

(ii) Once again, let us use equation (7). Now, if $H$ is convex, it is bounded above by the piecewise linear function taking values $1+\frac{y^{*}-1}{y^{*}} x$ for $x \in\left[0, y^{*}\right]$ and $-\frac{y^{*}}{y^{*}-1}$ $+\frac{y^{*}}{y^{*}-1} x$ for $x \in\left(y^{*}, 1\right]$. The result follows. A similar argument proves the result for $H$ concave.

\section{References}

Aczel J, Wagner C (1981) Rational decision making generalized. Math Rep Acad Sci Canada 3:138-142

Alesina A, Rosenthal H (1995) Partisan politics, divided government and the economy. Cambridge University Press, Cambridge

Alesina A, Rosenthal H (1996) A theory of divided government Econometrica 64(6):1311-1343

Barbera S (1977) The manipulation of social choice mechanisms that do not leave "too much" to chance. Econometrica 45(7):1573-1588

Barbera S (2006) An introduction to strategy-proof social choice functions. Social Choice Welfare 18(4): $619-653$

Barbera S (2011) Strategy-proof social choice. In: Arrow K, Suzumura K (eds) Handbook of social choice and welfare, vol 2. Kluwer, Dordrecht, pp 731-831

Bernheim D, Peleg D, Whinston M (1987) Coalition-proof Nash equilibria I: concepts. J Econ Theory 42:1-12

Bilodeau M (1994) Tax earmarking and separate school financing. J Public Econ 54:51-63

Border K, Jordan J (1983) Straightforward election, unanimity and phantom voters. Rev Econ Stud 50: $153-170$

Ching S (1995) What is the rationale for majority decisions? A characterization of pairwise majority rule. Mimeo

Ehlers L, Peters H, Storcken T (2004) Threshold strategy-proofness: on manipulability in large voting problems. Games Econ Behav 49:103-116

Gerber A, Ortuno-Ortin I (1998) Political compromise and endogenous formation of coalitions. Soc Choice Welfare 15:445-454

Gibbard A (1973) Manipulation of voting schemes. Econometrica 41:587-601

Goldie CM (1977) Convergence theorems for empirical Lorenz curves and their inverses. Adv Appl Probab 9:765-791

Hardy G, Littlewood J, Polya R (1952) Inequalities. Cambridge University Press, Cambridge

Holzman R (1990) An axiomatic approach to location on networks. Math Oper Res 15(3):553-563

Maskin E (1985) The theory of Nash implementation: a survey. In: Hurwicz L, Schmeidler D, Sonnenschein H (eds) Social goal and social organisation: volume in memory of Elisha Pazner. Cambridge University Press, Cambridge pp 173-204

Marchese C, Montefiori M (2010) Strategy versus sincerity in mean voting. J Econ Psychol. doi:10.1016/ j.joep.2010.12.003

Moulin H (1980) On strategy-proofness and single peakedness. Public Choice 35:437-455

Ordeshook PC (1986) Game theory and politics: an introduction. Cambridge University Press, Cambridge

Renault R, Trannoy A (2005a) Protection of minorities through the average voting rule. J Public Econ Theory 7(2):169-199

Renault R, Trannoy A (2005b) The Bayesian average voting game with a large population. Economie Publique/Public Economics, Issue in Honor of Louis-André Gérard-Varet, vol 17, pp 155-167

Satterthwaite M (1975) Strategy-proofness and Arrow's conditions: existence and correspondence theorems for voting procedures and social welfare functions. J Econ Theory 10:187-192

Sprumont Y (1995) Strategy-proof collective choice in economic and political environment. Can J Econ 18:68-107 